\title{
Neonatal intrahepatic cholestasis caused by citrin deficiency with no hepatic steatosis: a case report
}

\author{
Ryosuke Miyamoto ${ }^{1 *}$, Jun Sada ${ }^{1}$, Koki Ota ${ }^{2}$, Kenitiro Kaneko ${ }^{3}$, Hironori Kusano ${ }^{4}$, Yoshiteru Azuma ${ }^{1}$ and
} Akihisa Okumura ${ }^{1}$

\begin{abstract}
Background: Neonatal intrahepatic cholestasis caused by citrin deficiency (NICCD) is a common form of neonatal jaundice. Histopathological examination of the liver in patients with NICCD typically shows fatty liver, steatohepatitis, and liver fibrosis. Jaundice and fatty liver often improve by 1 year of age. We herein describe a girl who was diagnosed with NICCD based on an SLC25A13 mutation, although no fatty deposits were found on pathologic examination of the liver.

Case presentation: The patient in this case was a 3-month-old girl. At 2 months of age, she presented with jaundice, discolored stools, and poor weight gain and was found to have hyperbilirubinemia. Cholangiography revealed that she did not have biliary atresia. A laparoscopic liver biopsy was performed, and liver histopathology showed no fatty deposits. Genetic analysis revealed a compound heterozygous mutation in SLC25A13, and she was diagnosed with NICCD. She was given medium-chain triglyceride milk and gained weight. She resumed consumption of normal milk and breast milk, and her stool color improved. She was discharged at 4 months of age with adequate weight gain and a lower total bilirubin concentration. She was in good condition after discharge and showed normal development at the time of outpatient follow-up.
\end{abstract}

Conclusions: We experienced a case of NICCD in a patient without fatty liver. This case illustrates that the absence of hepatic steatosis in neonatal cholestasis does not rule out NICCD.

Keywords: Neonatal intrahepatic cholestasis caused by citrin deficiency, Fatty liver, Genetic analysis

\section{Background}

Neonatal intrahepatic cholestasis caused by citrin deficiency (NICCD) is a typical metabolic disorder that causes neonatal cholestasis. There are two clinical phenotypes of citrin deficiency: NICCD, which occurs from the neonatal to infantile period, and adult-onset type II citrullinemia (CTLN2), which occurs after puberty. In the period between these two forms, there is also a form

\footnotetext{
* Correspondence: ryosk.mymti@gmail.com

'Department of Pediatrics, Aichi Medical University School of Medicine, 1-1 Yazakokarimata, Nagakute, Aichi 480-1195, Japan

Full list of author information is available at the end of the article
}

called failure to thrive and dyslipidemia caused by citrin deficiency (FTTDCD), which is an adaptive and compensatory phase. The responsible gene is SLC25A13. The encoded protein, citrin, is an aspartate-glutamate carrier localized primarily in the mitochondrial inner membrane of the liver. This transport works as part of the malate aspartate shuttle. When aspartate is not transported into the cytoplasm due to citrin deficiency, the urea cycle is not levable to operate, and this causes citrulline levels to elevate. Loss of citrin function impairs urea and protein synthesis, aerobic glycolysis, glycogenesis, and energy metabolism, leading to a variety of

(c) The Author(s). 2021 Open Access This article is licensed under a Creative Commons Attribution 4.0 International License, which permits use, sharing, adaptation, distribution and reproduction in any medium or format, as long as you give appropriate credit to the original author(s) and the source, provide a link to the Creative Commons licence, and indicate if changes were made. The images or other third party material in this article are included in the article's Creative Commons licence, unless indicated otherwise in a credit line to the material. If material is not included in the article's Creative Commons licence and your intended use is not permitted by statutory regulation or exceeds the permitted use, you will need to obtain permission directly from the copyright holder. To view a copy of this licence, visit http://creativecommons.org/licenses/by/4.0/. The Creative Commons Public Domain Dedication waiver (http//creativecommons.org/publicdomain/zero/1.0/) applies to the data made available in this article, unless otherwise stated in a credit line to the data. 
symptoms. Histopathologic examination of the liver of children with NICCD generally shows fatty liver, steatohepatitis, hepatic fibrosis, and, rarely, cirrhosis. Cholestasis and fatty liver often improve by 1 year of age, but whether they later progress to CTLN2 remains to be determined. We herein describe a girl with NICCD diagnosed on the basis of variants in SLC25A13, although no fat deposition was seen upon pathological examination of the liver.

\section{Case presentation}

The patient was a 3-month-old girl born at 40 weeks of gestational age with a birth weight of $2600 \mathrm{~g}$. She was the first child of healthy non-consanguineous parents. She had no history of phototherapy for jaundice. Newborn Screening and regular 1-month checkup were unremarkable. At 2 months of age, the patient's mother noticed jaundice and discolored stool and visited a local clinic. The infant's weight gain had been insufficient during the last month. Her body weight remained around $4500 \mathrm{~g}$ despite the fact that she had been fully breastfed 8 to 10 times/day. She had icteric sclera and her skin was yellowish brown. Laboratory examinations showed a total bilirubin concentration of $9.74 \mathrm{mg} / \mathrm{dl}$, direct bilirubin concentration of $5.64 \mathrm{mg} / \mathrm{dl}$, and prothrombin time-international normalized ratio of 1.2. The patient was referred to our hospital after intravenous vitamin $\mathrm{K}$ infusion.

Although she was in good condition, yellowing of the sclera was evident and the skin was brownish in color. She excreted creamy grayish-white stools. Blood test data at the time of admission were AST $195 \mathrm{U} / \mathrm{L}$, ALT $43 \mathrm{U} / \mathrm{L}$, GGT $95 \mathrm{U} / \mathrm{L}$, albumin $3.7 \mathrm{~g} / \mathrm{dl}$, AFP $35898.7 \mathrm{ng} /$ $\mathrm{ml}$, T-chol $196 \mathrm{mg} / \mathrm{dl}$, and triglycerides $233 \mathrm{mg} / \mathrm{dl}$. At the last follow-up at 13 months of age, the data were AST $69 \mathrm{U} / \mathrm{L}$, ALT $29 \mathrm{U} / \mathrm{L}$, GGT $83 \mathrm{U} / \mathrm{L}$, albumin $4.5 \mathrm{~g} /$ $\mathrm{dl}$, total cholesterol $208 \mathrm{mg} / \mathrm{dl}$, and triglycerides $233 \mathrm{mg} /$ dl. And no evidence of Epstein-Barr virus, Cytomegalovirus, Hepatitis $A, B$, and $C$ virus was found in this case. Serum amino acid analysis showed a normal citrulline level of $39.9 \mathrm{nmol} / \mathrm{ml}$ and an elevated threonine concentration of $385.0 \mathrm{nmol} / \mathrm{ml}$. The threonine/serine ratio was within the normal range. Abdominal ultrasonography confirmed the presence of a gallbladder and the absence of the triangular cord sign in the hilar region, and 24-h biliary scintigraphy showed poor excretion. Biliary atresia was ruled out by cholangiography. We performed laparoscopic liver biopsy. Macroscopically, the liver was generally yellow due to cholestasis. Pathological examination demonstrated cholestasis and hydropic changes, no obvious inflammatory cell infiltration within the lobule, no evidence of fat deposition (Fig. 1a), and a mild ductular reaction in the margins of the portal area as

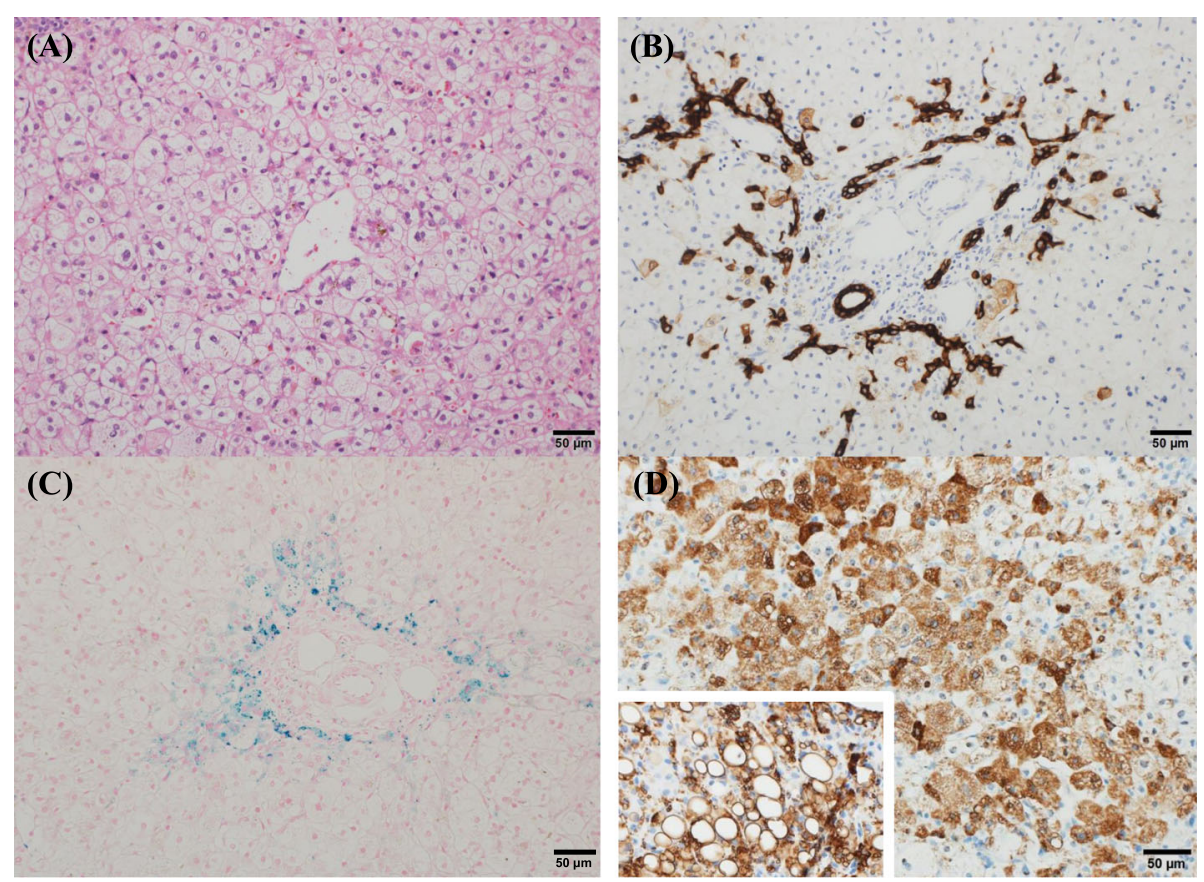

Fig. 1 Pathological findings of liver tissue. a Hepatocyte swelling is notable. Bile plugs are also present. There is no fat deposition in the hepatocytes. b Cytokeratin 7 immunostaining shows a mild ductular reaction in the rim of the portal area and a clear interlobular bile duct. $\mathbf{c}$ Deposits of hemosiderin are found in the hepatocytes around the portal area. $\mathbf{d}$ Adipophilin immunostaining shows that the cytoplasm of the hepatocytes is positive, but perivacuolar staining is not highlighted (inset, positive control of fatty liver sample) 
confirmed by cytokeratin 7 immunohistochemistry (Fig. 1b). The bile ducts were adequate in number, and the interlobular bile duct count/portal vein count ratio was about 0.7. Berlin blue staining revealed iron (hemosiderin) deposition in the periportal hepatocytes (Fig. 1c). Immunostaining for adipophilin, a fatty droplet-binding protein, showed positive staining in the cytoplasm of the hepatocytes; however, the perivacuolar areas were not clearly stained, indicating no evidence of steatosis (Fig. 1d).

Genetic analysis revealed compound heterozygous variants in SLC25A13: NM_014251.3, c.674C > A(p.Ser225*), and c.1801G > T(p.Glu601*). The infant's father and mother were found to be carriers of each mutation. She was diagnosed with NICCD and treated with ursodeoxycholic acid. Her bilirubin concentration gradually declined and her jaundice improved. Her stool color returned to normal. She was fed medium-chain triglyceride milk and her body weight increased. At 5 months of age, she resumed consumption of normal milk and breast milk, and her stool color improved. Her body weight exceeded $5000 \mathrm{~g}$, and her total bilirubin concentration decreased to around $2 \mathrm{mg} / \mathrm{dl}$. She was discharged from the hospital at 4 months of age. She was in good condition after discharge and showed normal psychomotor development at the last follow-up at 13 months of age.

\section{Discussion and conclusions}

NICCD is an autosomal recessive disease that causes intrahepatic cholestasis in newborns. There are two major categories of NICCD: one in which a positive newborn screening (high levels of galactose, methionine, and phenylalanine) reveals liver damage, and the other in which newborn screening is normal and the patient is diagnosed with prolonged jaundice and poor weight gain after 1 month of age [1]. The present case falls into the latter category.

NICCD is known to be often associated with fatty liver on pathological examination. Remarkably, however, fatty liver was not present in our patient. Kimura et al. [2] reported the pathological findings of 30 patients with NICCD; 29 patients showed fatty liver, and the fatty deposits were especially severe in 20 patients. Only one patient had no hepatic steatosis. However, detailed information on the genetic mutations, symptoms, and outcomes was not available [2]. Inui et al. [3] described an 8-year-old girl with an SLC25A13 mutation who showed no fatty changes of the liver by light microscopy. In this patient, very small fatty droplets were observed by electron microscopy [3]. Although we did not evaluate our patient by electron microscopy, no fatty deposits were observed upon immunostaining for adipophilin.
We consider that fatty changes of the liver were absent in our patient.

Komatsu et al. [4] reported that the expression of peroxisome proliferator-activated receptor alpha (PPAR $\alpha$ ), a master regulator of hepatic lipid metabolism, was significantly reduced in the liver tissue of patients with CTLN2. The expression of hepatic PPAR $\alpha$ was inversely correlated with the severity of steatosis and the citrin concentration, indicating that down-regulation of PPAR $\alpha$ was associated with fatty liver in patients with CTLN2. The expression of hepatic PPAR $\alpha$ should be further investigated in patients with NICCD to clarify the reason for the different severity of fatty liver.

At present, the genotype-phenotype correlation of SLC25A13 is unclear. We found a combination of two nonsense variants (S225X and E601X) in our patient. We presume that homozygous or compound heterozygous situations of nonsense variants can cause NICCD. For instance, homozygous or compound heterozygous situations of two mutations (c.851del4 and IVS11L1G > A) are caused both early- and late-onset citrin deficiency [5]. Although we did not find the same combination of variants in previous reports as in our patient, each variant caused phenotypes of both NICCD and CTLN2 [58]. We experienced a case of NICCD without fatty liver. NICCD should always be included in the differential diagnosis of cholestasis in newborns. This case suggests that NICCD should not be ruled out even if hepatic steatosis is not seen in newborns with cholestasis.

\section{Abbreviations}

NICCD: Neonatal intrahepatic cholestasis caused by citrin deficiency; CTLN2: Adult-onset type II citrullinemia; PPARa: Peroxisome proliferatoractivated receptor alpha

\section{Acknowledgments}

We are grateful to the doctors of the Department of Pediatrics at Nagoya City University for the genetic analysis.

\section{Authors' contributions}

RM wrote the first draft of the manuscript and contributed to the data interpretation. JS, KO, KK, HK, and YA reviewed and revised the manuscript. OA coordinated and supervised the data collection and critically reviewed the manuscript for important intellectual content. All authors approved the final manuscript as submitted and agree to be accountable for all aspects of the work.

\section{Funding}

This article received no specific grant from any funding agency.

\section{Availability of data and materials}

The data that support the findings of this case report are available from the corresponding author upon reasonable request.

\section{Declarations}

Ethics approval and consent to participate

All examinations and investigations in this case were approved by the institutional review board of Aichi Medical University (registration No. 2020H076). 


\section{Consent for publication}

Written informed consent that includes the publication of this report and accompanying images has been obtained from her father and mother for all educational and academic purposes, including the publication of this case report.

\section{Competing interests}

The authors declare that they have no competing interests.

\section{Author details}

'Department of Pediatrics, Aichi Medical University School of Medicine, 1-1 Yazakokarimata, Nagakute, Aichi 480-1195, Japan. ${ }^{2}$ Postgraduate Clinical Training Center, Aichi Medical University, Nagakute, Aichi, Japan.

${ }^{3}$ Department of Surgery, Aichi Medical University, Nagakute, Aichi, Japan. ${ }^{4}$ Department of Pathology, Kurume University School of Medicine, Kurume, Japan.

Received: 22 December 2020 Accepted: 13 May 2021

Published online: 18 May 2021

\section{References}

1. Ohura T, Kobayashi K, Tazawa Y, Abukawa D, Sakamoto O, Tsuchiya S, et al. Clinical pictures of 75 patients with neonatal intrahepatic cholestasis caused by citrin deficiency (NICCD). J Inherit Metab Dis. 2007;30(2):139-44.

2. Kimura A, Kage M, Nagata I, Mushiake S, Ohura T, Tazawa Y, et al. Histological findings in the livers of patients with neonatal intrahepatic cholestasis caused by citrin deficiency. Hepatol Res. 2010;40(4):295-303.

3. Inui A, Hashimoto T, Sogo T, Komatsu H, Saheki T, Fujisawa T. Chronic hepatitis without hepatic steatosis caused by citrin deficiency in a child. Hepatol Res. 2016;46(4):357-62.

4. Komatsu M, Kimura T, Yazaki M, Tanaka N, Yang Y, Nakajima T, et al. Steatogenesis in adult-onset type II citrullinemia is associated with downregulation of PPARalpha. Biochim Biophys Acta. 2015;1852(3):473-81.

5. Yamaguchi N, Kobayashi K, Yasuda T, Nishi I, lijima M, Nakagawa M, et al. Screening of SLC25A13 mutations in early and late onset patients with citrin deficiency and in the Japanese population: identification of two novel mutations and establishment of multiple DNA diagnosis methods for nine mutations. Hum Mutat. 2002;19(2):122-30.

6. Kobayashi K, Sinasac DS, lijima M, Boright AP, Begum L, Lee JR, et al. The gene mutated in adult-onset type II citrullinaemia encodes a putative mitochondrial carrier protein. Nat Genet. 1999;22(2):159-63.

7. Hayasaka K, Numakura C, Toyota K, Kimura T. Treatment with lactose (galactose)-restricted and medium-chain triglyceride-supplemented formula for neonatal intrahepatic cholestasis caused by citrin deficiency. JIMD Rep. 2012;2:37-44.

8. Togawa T, Sugiura T, Ito K, Endo T, Aoyama K, Ohashi K, et al. Molecular Genetic Dissection and Neonatal/Infantile Intrahepatic Cholestasis Using Targeted Next-Generation Sequencing. J Pediatr. 2016;171:e171-4.

\section{Publisher's Note}

Springer Nature remains neutral with regard to jurisdictional claims in published maps and institutional affiliations. 